\title{
ESTIMATES OF SOME PROBABILITIES IN MULTIDIMENSIONAL CONVEX RECORDS
}

Abstract. Convex records in Euclidean space are considered. We provide both lower and upper bounds on the probability $p_{n}(k)$ that in a sequence of random vectors $X_{1}, \ldots, X_{n}$ there are exactly $k$ records.

1. Introduction. Records on a line have received a good deal of attention in the last thirty years. The reader may be referred to Nevzorov [8] and Resnick [9] for some recent studies on this model. There has been a trend in the past few years to move away from the standard model and to consider either records for random elements in a partially ordered space or convex records for random vectors in $d$-dimensional Euclidean space (see $[3,9]$ ).

The purpose of this paper is to investigate the case of convex records defined as follows. Suppose independent and identically distributed random vectors $X_{i}=\left(X_{i 1}, \ldots, X_{i d}\right), i=1,2, \ldots$, are observed. Define random variables $L(n)$ as follows: $L(0)=0, L(1)=1$, and $L(n+1)=\min \{i$ : $\left.X_{i} \notin \operatorname{conv}\left\{X_{1}, \ldots, X_{L(n)}\right\}\right\}, n>1$, where $\operatorname{conv}\left\{X_{1}, \ldots, X_{n}\right\}$ is the convex hull of $X_{1}, \ldots, X_{n}$. In addition, define $N(n)=\max \{k: L(k) \leq n\}$. Throughout the paper $L(k)$ is called the time of the $k$ th convex record. Then $N(n)$ is the cardinality of the set of convex records which occur up to time $n$. Further, for $k=d+1, \ldots, n$, we set

$$
p_{n}(k)=\operatorname{Pr}\{N(n)=k\} \quad \text { and } \quad q_{n}(k)=\operatorname{Pr}\{L(k)=n\} .
$$

The problem of convex records dates back to the nineteenth century. The case of randomly chosen points within the $d$-dimensional unit ball was considered for the first time by Sylvester (see [6]). He posed the problem of calculation of $p_{d+2}(d+1)$. An important contribution to solving Sylvester's problem was made by Blaschke and Hostinsky but it was Kingman [7] who

1991 Mathematics Subject Classification: Primary 60D05; Secondary 60E05, 60G70.

Key words and phrases: geometric probability, convex records in Euclidean space, estimates of probabilities. 
obtained the exact formula for $p_{d+2}(d+1)$. The first result on the asymptotic distribution of the number of vertices of the convex hull of $n$ randomly chosen points, say $N_{n}$, was given by Rényi and Sulanke (cf. [6]). Groeneboom [5] continued the work on this problem and obtained the asymptotic distribution of $N_{n}$ as $n$ tends to infinity. Bárany and Füredi [1] also examined the limiting behaviour of $\operatorname{Pr}\left\{N_{n}=k\right\}$ as either $k$ or $d$ goes to infinity.

In this article we are concerned with the distribution of the random variables $L(n)$ and $N(n)$ for $n=d+1, d+2, \ldots$ In Section 2 we present the exact formula for $p_{n}(d+1)$ and provide both lower and upper bounds for $p_{n}(k)$ and $q_{n}(k)$ for $k \geq d+2$ in the case of convex records within a unit ball. Section 3 concerns some numerical study.

2. The main results. We first set up the basic notation and assumptions. Suppose that $X_{1}, X_{2} \ldots$ are modeled as independent observations in $\mathbb{R}^{d}$ with a common density $f$ and that $K, K=\{x: f(x)>0\}$, is a bounded convex subset of $\mathbb{R}^{d}$. Let $C\left(x_{1}, \ldots, x_{k}\right)$ denote the $k$-neighbourly polytope whose vertices are $x_{1}, \ldots, x_{k}$, and let $F$ be the distribution of $X_{1}$. Define

$M_{k}=\sup \left\{\int_{C\left(x_{1}, \ldots, x_{k}\right)} d F(x): C\left(x_{1}, \ldots, x_{k}\right) \subset K\right\}, \quad k=d+2, d+3, \ldots$

The following proposition gives both upper and lower bounds on the probability $p_{n}(k)$ that in the sequence $X_{1}, \ldots, X_{n}$ there are exactly $k$ records.

Proposition 1. With the notation given above, define

$$
\alpha_{k}=\int_{\mathbb{R}^{d(d+1)}}\left(\int_{C\left(x_{1}, \ldots, x_{d+1}\right)} d F(x)\right)^{k} d F\left(x_{1}\right) \ldots d F\left(x_{d+1}\right) .
$$

Then:

(i) $p_{n}(d+1)=\alpha_{n-d-1}$.

(ii) For $d+2 \leq k \leq n$,

$$
\begin{aligned}
& \left(\begin{array}{c}
n-d-1 \\
k-d-1
\end{array}\right) \prod_{i=d+2}^{k-1}\left(1-M_{i}\right)\left(\alpha_{n-k}-\alpha_{n-k+1}\right) \leq p_{n}(k) \\
& \quad \leq \sum_{i=0}^{n-k} \sum_{j=0}^{k-d-1}\left(\begin{array}{c}
n-d-i-2 \\
k-d-2
\end{array}\right)\left(\begin{array}{c}
k-d-1 \\
j
\end{array}\right)(-1)^{j} M_{k}^{n-k-i} \alpha_{i+j},
\end{aligned}
$$

where $\prod_{i=d+2}^{d+1}\left(1-M_{i}\right)$ is 1 by convention.

Proof. Using standard arguments it is possible to show that for $k=$ $d+1, \ldots, n$, 
(2) $\quad p_{n}(k)=\sum_{\left(n_{i}\right) \in \mathcal{N}} \int_{\mathbb{R}^{d(d+1)}}\left(\int_{C_{d+1}} d F(x)\right)^{n_{1}} d F\left(x_{1}\right) \ldots d F\left(x_{d+1}\right)$

$$
\begin{aligned}
& \times \int_{\mathbb{R}^{d} \backslash C_{d+1}}\left(\int_{C_{d+2}} d F(x)\right)^{n_{2}} d F\left(x_{d+2}\right) \times \ldots \\
& \times \int_{\mathbb{R}^{d} \backslash C_{k-1}}\left(\int_{C_{k}} d F(x)\right)^{n_{k-d}} d F\left(x_{k}\right)
\end{aligned}
$$

with

$$
\mathcal{N}=\left\{\left(n_{1}, \ldots, n_{k-d}\right): k+n_{i}=n \text { and } n_{i} \geq 0 \text { for all } i\right\} .
$$

Here $C_{n}=\operatorname{conv}\left\{x_{1}, \ldots, x_{n}\right\}$. Hence the result for $k=d+1$ follows directly. To prove (ii) we use the following estimates:

$$
\int_{C_{i}} d F(x) \leq \int_{C_{k}} d F(x) \quad \text { for } i \leq k,
$$

$$
1-M_{k} \leq \int_{\mathbb{R}^{d} \backslash C_{k}} d F(x),
$$

$$
\int_{C_{k}} d F(x) \leq M_{k}
$$

$$
\int_{\mathbb{R}^{d} \backslash C_{k}} d F(x) \leq \int_{\mathbb{R}^{d} \backslash C_{d+1}} d F(x) \quad \text { for } k \geq d+1 .
$$

Combining (3) and (4) yields

$$
\begin{aligned}
p_{n}(k) \geq & \# \mathcal{N} \int_{\mathbb{R}^{d(d+1)}} d F(x)\left(\int_{C_{d+1}} d F(x)\right)^{n-k} \\
& \times\left(1-\int_{C_{d+1}} d F(x)\right) d F\left(x_{1}\right) \ldots d F\left(x_{d+1}\right),
\end{aligned}
$$

where $\# \mathcal{N}$ denotes the cardinality of $\mathcal{N}$. Next use (5) and (6) to get

$$
\begin{aligned}
p_{n}(k) \leq & \sum_{\left(n_{i}\right) \in \mathcal{N}} \prod_{i=d+2}^{k} M_{i}^{i-d} \int_{\mathbb{R}^{d(d+1)}}\left[\int_{C_{d+1}} d F(x)\right]^{n_{1}} \\
& \times\left[1-\int_{C_{d+1}} d F(x)\right]^{k-d-1} d F\left(x_{1}\right) \ldots d F\left(x_{d+1}\right) \\
\leq & \sum_{i=0}^{n-k} \# \mathcal{N}_{i} \cdot M_{k}^{n-k-i} \sum_{j=0}^{k-d-1}\left(\begin{array}{l}
n-d-1 \\
k-d-1
\end{array}\right)(-1)^{j} \alpha_{i+j},
\end{aligned}
$$

where $\mathcal{N}_{i}=\left\{\left(n_{2}, \ldots, n_{k-d}\right): k+i+n_{s}=n\right.$ and $n_{s} \geq 0$ for all $\left.s\right\}$. Now, 
application of a combinatorial lemma (see [2], Chapter II, 5) to both (7) and (8) yields the desired result.

The next proposition gives worse estimates than those of Proposition 1 but which are more useful to derive asymptotic results.

Proposition 2. Under the assumptions of Proposition 1,

(9) $\quad\left(\begin{array}{l}n-d-1 \\ k-d-1\end{array}\right) \prod_{i=d+1}^{k-1}\left(1-M_{i}\right) \alpha_{n-k} \leq p_{n}(k) \leq\left(\begin{array}{l}n-d-1 \\ k-d-1\end{array}\right) M_{k}^{n-k}$,

for $k=d+2, \ldots, n$.

Proof. The proof follows along the same lines as in Proposition 1 and is left to the reader.

The above propositions allow us to estimate some probabilities in the multidimensional model of convex records if we are able to obtain the exact form of $\alpha_{k}$ and $M_{k}$ for $k=1,2, \ldots$ Consider the case of independent observations from the unit ball on the plane; that is, $X_{1}$ has a uniform distribution over $K$, where $K=\left\{(x, y): x^{2}+y^{2} \leq 1\right\}$. Henceforth, we write $n ! !=3 \cdot 5 \cdot \ldots \cdot n$ for $n$ odd and $2 \cdot 4 \cdot \ldots \cdot n$ for $n$ even. We also put $0 ! !=1$ and $(-1) ! !=1$.

THEOREM 3. Let $p_{n}(k)$ be the probability that in a sequence of $n$ randomly chosen points from the unit ball on the plane there are exactly $k$ convex records. Suppose that $d=2$. Then

$$
\begin{aligned}
\alpha_{k}= & \frac{3 \cdot 2^{k+6}}{(k+2)^{2}(k+3) \pi^{k+4}} \sum_{i=0}^{(k-1) / 2}\left(\begin{array}{c}
k \\
2 i
\end{array}\right) \sum_{s=0}^{(k-1) / 2-i}\left(\begin{array}{c}
(k-1) / 2-i \\
s
\end{array}\right)(-1)^{s} \\
& \times \frac{(3 k-1+2 s+5) ! !(2 i-1) ! !}{(k+2 s+2 i+3)[(3 k-1) / 2+s+i+3] !} \cdot 0.5^{(3 k-1) / 2+s+i+3} \\
= & \frac{3 \cdot 2^{-2 k+1}}{(k+2)^{2}(k+3) \pi^{k}} \sum_{i=0}^{k / 2}\left(\begin{array}{c}
k \\
2 i
\end{array}\right) \frac{(k+2 i+1) ! !(k-2 i) ! ! 4^{-i}}{(k+1) k \ldots(k / 2-i+1)(k / 2-i) !} \\
& \times \sum_{s=0}^{i}\left(\begin{array}{c}
i \\
s
\end{array}\right)\left(\begin{array}{c}
2(k+s-i+1) \\
k+s-i+1
\end{array}\right)(-1)^{s} 4^{-s} \quad \text { for } k=0,2,4, \ldots
\end{aligned}
$$

and conclusions (i) and (ii) of Proposition 1 hold.

Proof. By Proposition 1, it is enough to derive the exact form of $\alpha_{k}$ and $M_{k}$. Since $M_{k}=(2 \pi / k) \sin (k / 2 \pi)$ (cf. [10], Problem 57), we only have 
to evaluate the integral

$$
\alpha_{k}=\left(\pi r^{2}\right)^{-(k+3)} \int_{K(r)^{2}}\left|C\left(x_{1}, x_{2}, x_{3}\right)\right|^{k} d x_{1} d x_{2} d x_{3},
$$

where $K(r)=\left\{(x, y): x^{2}+y^{2} \leq r^{2}\right\}, x_{i}=\left(x_{i 1}, x_{i 2}\right)$ for $i=1,2,3$, $C\left(x_{1}, x_{2}, x_{3}\right)=\operatorname{conv}\left\{x_{1}, x_{2}, x_{3}\right\}$ and $|\cdot|$ stands for the Lebesgue measure. Direct calculations show that

$$
\begin{aligned}
\text { (10) } \alpha_{k}= & \left(\pi r^{2}\right)^{-(k+3)} \int_{[0,2 \pi]^{3}} \int_{[0, r]^{3}}\left(0.5 \mid u w \cdot \sin \left(\theta_{2}-\theta_{3}\right)+w z \cdot \sin \left(\theta_{1}-\theta_{2}\right)\right. \\
& \left.+u z \cdot \sin \left(\theta_{3}-\theta_{1}\right) \mid\right)^{k} u w z d u d w d z d \theta_{1} d \theta_{2} d \theta_{3} .
\end{aligned}
$$

Unfortunately, for general $k$, it seems difficult to obtain $\alpha_{k}$ explicity from (10) so the technique similar to that of Crafton (see [7], Chapter 2) is proposed.

Letting $a_{k}(r)$ denote $\left(\pi r^{2}\right)^{k+3} \alpha_{k}(r)$, and $K(r, r+\delta)$ denote $K(r+\delta) \backslash$ $K(r)$, we calculate the derivative $a_{k}^{\prime}(r)$. First note that

$$
a_{k}(r+\delta)-a_{k}(r)=3 \int_{K(r, r+\delta)} d x_{1} \int_{K(r)^{2}}\left|C\left(x_{1}, x_{2}, x_{3}\right)\right|^{k} d x_{1} d x_{2} d x_{3}+o(\delta) .
$$

This follows from the definition of $a_{k}(r)$ and the estimates

$$
\left.\int_{K(r, r+\delta)^{2}} d x_{1} d x_{2} \int_{K(r)} C\left(x_{1}, x_{2}, x_{3}\right)\right|^{k} d x_{3} \leq\left(\pi r^{2}\right)^{k}\left[\pi(r+\delta)^{2}-\pi r^{2}\right]^{2}=o(\delta)
$$

and

$$
\int_{K(r, r+\delta)^{3}}\left|C\left(x_{1}, x_{2}, x_{3}\right)\right|^{k} d x_{1} d x_{2} d x_{3} \leq o(\delta) .
$$

Now, after the transformation $x_{11}=u \cos \phi$ and $x_{12}=u \sin \phi$, we can obtain

$$
\begin{aligned}
a_{k}(r+ & \delta)-a_{k}(r) \\
& =3 \int_{r}^{r+\delta} u d u \int_{0}^{\pi} d \phi \int_{K(r)^{2}}\left|C\left((u \cos \phi, u \sin \phi), x_{2}, x_{3}\right)\right|^{k} d x_{2} d x_{3}+o(\delta) .
\end{aligned}
$$

Thus, by dominated convergence,

$$
a_{k}^{\prime}(r)=6 \pi r \int_{K(r)^{2}}\left|C\left(x_{1}, x_{2}, x_{3}\right)\right|^{k} d x_{2} d x_{3},
$$

$x_{1}$ being any point of the boundary of $K(r)$. Further, applying the transformation $x_{21}=a \cos \theta, x_{22}=a \sin \theta, x_{31}=b \cos \phi$ and $x_{32}=b \sin \phi$, we 
get

$$
\begin{aligned}
& \text { (12) } \int_{K(r)^{2}}\left|C\left(x_{1}, x_{2}, x_{3}\right)\right|^{k} d x_{2} d x_{3} \\
& =\int_{0}^{\pi} \int_{0}^{\pi} \int_{0}^{2 r \sin \theta} \int_{0}^{2 r \cos \phi}(0.5 \cdot a b \sin |\theta-\phi|)^{k} a b d a d b d \theta d \phi=\frac{2^{k+4}}{(k+2)^{2}} r^{2 k+4} \gamma_{k},
\end{aligned}
$$

where

$$
\gamma_{k}=\int_{0}^{1} \int_{0}^{1} \sin ^{k+2} \theta \sin ^{k+2} \phi \sin ^{k}|\theta-\phi| d \theta d \phi .
$$

Combining (11) and (12), we have

$$
\alpha_{k}=\frac{3 \cdot 2^{k+4}}{(k+2)^{2}(k+3) \pi^{k+2}} \gamma_{k}
$$

By Appendix 1, this yields the desired result.

Now we formulate some asymptotic results dealing with $p_{n}(k)$ as $n$ tends to infinity.

TheOREM 4. Suppose that the conditions of Theorem 1 hold. Then for $k=4,5, \ldots$,

(i) $\lim _{n \rightarrow \infty} p_{n}(k)\left[M_{k}^{n-k}\right]^{-1} \leq\left[-\ln M_{k}\right]^{3-k}$,

(ii) $\lim _{n \rightarrow \infty} p_{n}(k)\left[\left(\frac{1}{\pi}\right)^{n-k} n^{4}\right]^{-1} \geq \frac{12}{\pi} \prod_{i=3}^{k-1}\left(1-M_{i}\right)(\ln \pi)^{3-k}$.

Proof. We apply Proposition 2. In what follows we write $f(x) \sim g(x)$ as $x \rightarrow \alpha$ iff $\lim _{x \rightarrow \alpha}(f(x) / g(x))=1$, where $\alpha \in \mathbb{R} \cup\{-\infty, \infty\}$. Using the well-known asymptotic formula $\Gamma(x+1) \sim x^{x} 2 \pi x e^{-x}$ as $x \rightarrow \infty$, we have

$$
\left(\begin{array}{l}
n-3 \\
k-3
\end{array}\right) \sim(n-3)^{k-3}[(k-3) !]^{-1} \quad \text { as } n \rightarrow \infty
$$

Hence

$$
p_{n}^{*}(k) \leq p_{n}(k) \leq p_{n}^{* *}(k)
$$

where

$$
p_{n}^{*}(k) \sim(n-3)^{k-3} \prod_{i=3}^{k-1}\left(1-M_{i}\right) \alpha_{n-k}[(k-3) !]^{-1}
$$

and

$$
p_{n}^{* *}(k) \sim(n-3)^{k-3} M_{k}^{n-k}[(k-3) !]^{-1} M_{k}^{n-k}\left[-\ln M_{k}\right]^{3-k},
$$

which completes the proof of (i). Here we use the fact that $x^{n} \exp (-b x) \sim$ $n ! b^{-n} \exp (-b x)$ as $x \rightarrow \infty$ provided $b>0$ and $n=0,1,2, \ldots$ 
To prove (ii), we note that

$$
\alpha_{k} \geq \frac{3 \cdot 2^{k+4}}{(k+2)^{2}(k+3) \pi^{k+3}} 2^{-k-3} B((k+3) / 2,0.5)
$$

where $B(x, y)=\Gamma(x) \Gamma(y) / \Gamma(x+y)$ (for the proof see Appendix 2). Consequently,

$$
p_{n}^{*}(k) \sim \frac{12}{\pi(\ln \pi)^{k-3}} \prod_{i=3}^{k-1}\left(1-M_{i}\right) \pi^{-(n-k)} n^{-4} .
$$

This establishes Theorem 4.

It is of interest how to improve the results of Theorems 3 and 4 . This might be done by evaluating mixed moments of the random variables $\left|C\left(x_{1}, x_{2}, x_{3}\right)\right|$ and $\left|C\left(x_{1}, \ldots, x_{4}\right)\right|$, namely,

$$
\int_{K^{4}}\left|C\left(x_{1}, x_{2}, x_{3}\right)\right|^{n_{1}}\left|C\left(x_{1}, x_{2}, x_{3}, x_{4}\right)\right|^{n_{2}} d x_{1} \ldots d x_{4},
$$

where $n_{1}, n_{2}=0,1,2, \ldots$ How to do this remains an open question.

Several corollaries are readily available from Theorem 3 or 4 . One of them gives an estimate for the rate of vanishing of the probability that in a sequence of randomly chosen points in the unit ball on the plane the $k$ th record occurs on the $n$th position.

Corollary 1. If $X_{1}, \ldots, X_{n}$ are independent uniformly distributed vectors over the 2-dimensional unit ball, then $q_{n}(k)$, defined by $(1)$, satisfies for $k=4,5, \ldots$,

$$
\lim _{n \rightarrow \infty} q_{n}(k)\left[M_{k-1}^{n}\right]^{-1} \leq M_{k-1}^{1-k}\left[-\ln M_{k-1}\right]^{k-4},
$$

where $M_{k}=(k / 2 \pi) \sin (2 \pi / k)$.

Proof. The proof is straightforward. It follows from Theorem 4 and the fact that

$$
\begin{aligned}
q_{n}(k) & =\operatorname{Pr}(L(k)=n)=\operatorname{Pr}(N(n-1)<k)-\operatorname{Pr}(N(n)<k) \\
& =\sum_{i=3}^{k-1}\left(p_{n-1}(i)-p_{n}(i)\right) .
\end{aligned}
$$

The cases of other convex bodies in $\mathbb{R}^{2}$ can be analyzed in a similar fashion but this is beyond the scope of the present work. Some extensions to higher dimensions are also possible. The main difficulty is to obtain the explicit form of $M_{k}$. Below we present some results for points randomly chosen in the $d$-dimensional unit ball. Even in this case the exact formula for $M_{k}$ is still unknown in the literature. However, there are a few estimates available. The simplest one is given by Ekeles' inequality, 


$$
M_{k} \leq k 2^{-d}
$$

where $k \geq 1$ and $d \geq 2$ (see [1]). Hence, (14) and Proposition 2 yield

COROLlary 2. Let $p_{n}(k, d)$ be the probability that among $n$ randomly chosen points from the d-dimensional unit ball there are exactly $k$ records. Then

$$
\lim _{n \rightarrow \infty} p_{n}(k, d)\left\{\left(k 2^{-d}\right)^{n-k}\right\}^{-1} \leq[d \ln 2-\ln k]^{1-d-k}
$$

provided $k<2^{d}$.

3. Numerical study. Herein the upper and lower estimates of $p_{n}(k)$ derived in Theorem 1 will be referred to as $p_{n}^{*}(k)$ and $p_{n}^{* *}(k)$, respectively. In order to check how precise the estimates are, we performed some numerical computations. Table 1 presents the results. For fixed $k>3$, the lower estimate is $p_{n}^{* *}(k)$ while the upper one is

$$
\min \left(p_{n}^{* *}(k), 1-p_{n}(3)-p_{n}^{*}(i)\right) .
$$

For instance, $p_{5}(3)=0.9499$ and $0.1288 \leq p_{5}(4) \leq 0.654$.

\section{TABLE 1}

\begin{tabular}{|c|c|c|c|c|c|}
\hline$n k$ & 3 & 4 & 5 & 6 & 7 \\
\hline 4 & $9.388 \mathrm{E}-02$ & $9.261 \mathrm{E}-01$ & & & \\
\hline \multirow[t]{2}{*}{5} & $9.499 \mathrm{E}-03$ & $6.540 \mathrm{E}-01$ & $8.617 \mathrm{E}-01$ & & \\
\hline & & $1.288 \mathrm{E}-01$ & $3.365 \mathrm{E}-01$ & & \\
\hline \multirow[t]{2}{*}{6} & $1.606 \mathrm{E}-03$ & $4.242 \mathrm{E}-01$ & $9.747 \mathrm{E}-01$ & $8.053 \mathrm{E}-01$ & \\
\hline & & $2.368 \mathrm{E}-02$ & $7.018 \mathrm{E}-02$ & $8.184 \mathrm{E}-02$ & \\
\hline \multirow[t]{2}{*}{7} & $3.208 \mathrm{E}-04$ & $2.714 \mathrm{E}-01$ & $9.945 \mathrm{E}-01$ & $9.973 \mathrm{E}-01$ & $7.554 \mathrm{E}-01$ \\
\hline & & $5.139 \mathrm{E}-03$ & $1.721 \mathrm{E}-01$ & $2.276 \mathrm{E}-02$ & $1.416 \mathrm{E}-02$ \\
\hline \multirow[t]{2}{*}{8} & $7.181 \mathrm{E}-05$ & $1.730 \mathrm{E}-01$ & $9.987 \mathrm{E}-01$ & $9.940 \mathrm{E}-01$ & $9.870 \mathrm{E}-01$ \\
\hline & & $1.245 \mathrm{E}-03$ & $4.669 \mathrm{E}-03$ & $6.975 \mathrm{E}-03$ & $4.921 \mathrm{E}-03$ \\
\hline \multirow[t]{2}{*}{9} & $1.746 \mathrm{E}-05$ & $1.102 \mathrm{E}-01$ & $9.997 \mathrm{E}-01$ & $9.983 \mathrm{E}-01$ & $9.960 \mathrm{E}-01$ \\
\hline & & $3.261 \mathrm{E}-04$ & $1.357 \mathrm{E}-03$ & $2.271 \mathrm{E}-03$ & $1.810 \mathrm{E}-03$ \\
\hline \multirow[t]{2}{*}{10} & $4.520 \mathrm{E}-06$ & $7.016 \mathrm{E}-02$ & $9.999 \mathrm{E}-01$ & $9.995 \mathrm{E}-01$ & $9.987 \mathrm{E}-01$ \\
\hline & & $9.057 \mathrm{E}-05$ & $4.148 \mathrm{E}-04$ & $7.701 \mathrm{E}-04$ & $6.875 \mathrm{E}-04$ \\
\hline \multirow[t]{2}{*}{20} & $3.379 \mathrm{E}-11$ & $7.673 \mathrm{E}-04$ & $2.313 \mathrm{E}-01$ & 1 & 1 \\
\hline & & $1.149 \mathrm{E}-09$ & $1.020 \mathrm{E}-08$ & $3.884 \mathrm{E}-08$ & $7.331 \mathrm{E}-08$ \\
\hline \multirow[t]{2}{*}{30} & $9.158 \mathrm{E}-16$ & $8.390 \mathrm{E}-06$ & $2.324 \mathrm{E}-02$ & 1 & 1 \\
\hline & & $4.405 \mathrm{E}-14$ & $5.834 \mathrm{E}-13$ & $3.333 \mathrm{E}-12$ & $9.816 \mathrm{E}-12$ \\
\hline \multirow[t]{2}{*}{40} & $4.105 \mathrm{E}-20$ & $9.174 \mathrm{E}-08$ & $1.986 \mathrm{E}-03$ & $8.599 \mathrm{E}-01$ & 1 \\
\hline & & $2.555 \mathrm{E}-18$ & $4.503 \mathrm{E}-17$ & $3.453 \mathrm{E}-16$ & $1.376 \mathrm{E}-15$ \\
\hline \multirow[t]{2}{*}{50} & $2.412 \mathrm{E}-24$ & $1.003 \mathrm{E}-09$ & $1.566 \mathrm{E}-04$ & $2.116 \mathrm{E}-01$ & 1 \\
\hline & & $1.842 \mathrm{E}-22$ & $4.058 \mathrm{E}-21$ & $3.904 \mathrm{E}-20$ & $1.964 \mathrm{E}-19$ \\
\hline \multirow[t]{2}{*}{100} & $7.023 \mathrm{E}-30$ & $1.568 \mathrm{E}-19$ & $2.915 \mathrm{E}-10$ & $7.008 \mathrm{E}-05$ & $3.071 \mathrm{E}-01$ \\
\hline & & $1.497 \mathrm{E}-25$ & $2.622 \mathrm{E}-24$ & $2.930 \mathrm{E}-23$ & $1.200 \mathrm{E}-22$ \\
\hline
\end{tabular}

Exact values of $p_{n}(3), n \geq 4$, and lower/upper estimates of $p_{n}(k)$, for $k=3,4,5,6,7$, in the case of convex records within the unit ball on the plane 
Appendix 1. In what follows we calculate

(A1) $\gamma_{k}=\int_{0}^{\pi} \int_{0}^{\pi} \sin ^{k+2} \theta \sin ^{k+2} \phi \sin ^{k}|\theta-\phi| d \phi d \theta=2 \sum_{i=0}^{k}\left(\begin{array}{c}k \\ i\end{array}\right)(-1)^{i} a_{i k}$,

with

$$
a_{i k}=\int_{0}^{\pi} \sin ^{k+i+2} \theta \cos ^{k-i} \theta \int_{\theta}^{\pi} \sin ^{2 k+2-i} \phi \cos ^{i} \phi d \phi d \theta .
$$

First suppose that $k-i$ is odd. Applying the formula

$$
\int_{0}^{\theta} \sin ^{a} u \cos ^{2 b+1} u d u=\sin ^{a+1} \theta \sum_{j=0}^{b}\left(\begin{array}{l}
b \\
j
\end{array}\right)(-1)^{j} \frac{\sin ^{2 j} \theta}{a+2 j+1},
$$

for $a \geq 0$ and $b=1,2, \ldots$, we have

$$
\begin{aligned}
a_{i k}= & \sum_{j=0}^{(k-i-1) / 2}\left(\begin{array}{c}
(k-i-1) / 2 \\
j
\end{array}\right) \frac{(-1)^{j}}{k+i+2 j+3} \\
& \times \int_{0}^{\pi} \sin ^{3 k+2 j+5} \theta \cos ^{i} \theta d \theta \\
= & \frac{1+(-1)^{i}}{2} \sum_{j=0}^{(k-i-1) / 2}\left(\begin{array}{c}
(k-i-1) / 2 \\
j
\end{array}\right) \frac{(-1)^{j}}{k+i+2 j+3} \\
& \times B\left(\frac{3 k+2 j+6}{2}, \frac{i+1}{2}\right) .
\end{aligned}
$$

Here we use the formula

$$
\int_{0}^{\pi} \sin ^{p} x \cos ^{q} x d x=\frac{1+(-1)^{q}}{2} B\left(\frac{p+1}{2}, \frac{q+1}{2}\right), \quad p, q>0
$$

(see [4], 3.427.1).

Now consider the case of $k-i$ even and $k$ odd. Then

$$
\begin{aligned}
a_{i k}= & -\sum_{j=0}^{(i-1) / 2}\left(\begin{array}{c}
(i-1) / 2 \\
j
\end{array}\right) \frac{(-1)^{j}}{2 k+3+2 j-i} \\
& \times \int_{0}^{\pi} \sin ^{3 k+2 j+5} \theta \cos ^{k-i} \theta d \theta \\
= & -\sum_{j=0}^{(i-1) / 2}\left(\begin{array}{c}
(i-1) / 2 \\
j
\end{array}\right) \frac{(-1)^{j}}{2 k+3+2 j-i}
\end{aligned}
$$




$$
\times B\left(\frac{3 k+2 j+6}{2}, \frac{k-i+1}{2}\right) .
$$

To complete the proof we suppose both $k$ and $i$ are even. Since we have

$$
\begin{aligned}
& \int_{0}^{\pi} \cos ^{m} x \sin ^{2 n} x d x \\
& =-\frac{\cos ^{m+1} \theta}{2 n+1}\left(\sum_{k=0}^{n-1} \frac{(2 n+1)(2 n-1) \ldots(2 n-2 k+1)}{(2 n+m)(2 n+m-2) \ldots(2 n+m-2)} \sin ^{2 n-2 k-1} \theta\right) \\
& \quad+\frac{(2 n-1) !}{(2 n+m)(2 n+m-2) \ldots(m+2)} \int_{0}^{\theta} \cos ^{m} x d x
\end{aligned}
$$

(see [4], 2.414), integration by parts gives

$$
\begin{aligned}
a_{i k}= & \frac{(k+i+1) ! !}{(2 k+2) 2 k \ldots(k-i+2)} \\
& \times \int_{0}^{\pi} \sin ^{2 k+2-i} \theta \cos ^{i} \theta \int_{0}^{\theta} \cos ^{k-i} x d x d \theta \\
= & \frac{(k+i+1) ! !}{(2 k+2) 2 k \ldots(k-i+2)} \\
& \times \sum_{s=0}^{i / 2}\left(\begin{array}{c}
i / 2 \\
s
\end{array}\right)(-1)^{s} \frac{(k-i-1) ! ! \pi^{2}}{[(k-i) / 2] ! \cdot(k+1+s-i / 2) !^{2}} \\
& \times(2 k-i+2 s+2) ! ! 2^{2 k+3-i+2 s+(k-i) / 2} .
\end{aligned}
$$

Here we use (2.415.1), (3.518.1), and (6.339.2) of [4]. Now from (A1)-(A4) and a little algebra one can obtain

$$
\begin{aligned}
\gamma_{k}= & 4 \pi \sum_{i=0}^{(k-1) / 2}\left(\begin{array}{c}
k \\
2 i
\end{array}\right) \sum_{s=0}^{(k-1) / 2-i}\left(\begin{array}{c}
(k-1) / 2-i \\
s
\end{array}\right)(-1)^{s} \frac{(3 k-1+2 s+5) ! !}{(k+2 s+2 i+3)} \\
& \times \frac{(2 i-1) ! !}{[(3 k-1) / 2+s+i+3] !}\left(\frac{1}{2}\right)^{(3 k-1) / 2+s+i+3} \quad \text { for } k=1,3,5, \ldots, \\
= & 2^{-3(k+1)} \pi^{2} \sum_{i=0}^{k / 2}\left(\begin{array}{c}
k \\
2 i
\end{array}\right) \frac{(k+2 i+1) ! !(k-2 i-1) ! !}{(k+1) k \ldots(k / 2-i+1)(k / 2-i) !} \cdot 4^{i} \\
& \times \sum_{s=0}^{i}\left(\begin{array}{l}
i \\
s
\end{array}\right)\left(\begin{array}{c}
2(k+s-i+1) \\
k+s-i+1
\end{array}\right)(-1)^{s} 4^{-s} \quad \text { for } k=0,2,4, \ldots
\end{aligned}
$$

This completes the proof. 
Appendix 2. Observe that

$$
\begin{aligned}
\gamma_{k} & \geq 2 \int_{0}^{\pi} \sin ^{k+2} \theta \int_{\pi / 2}^{\pi} \sin ^{k+2} \phi \sin ^{k}(\phi-\theta) d \phi d \theta \\
& \geq \int_{\pi / 2}^{\pi} \sin ^{k+2} \phi d \phi \int_{\pi / 4}^{\pi / 2} \sin ^{k+2} \phi \cos ^{k}(\theta) d \theta \\
& \geq 2 \int_{0}^{\pi / 2} \sin ^{k+2} \phi d \phi 2^{-k-2} \int_{0}^{\pi / 4} \sin ^{k+1} 2 \theta d \theta=2^{-k-3} B[(k+3) / 2,1 / 2]^{2} .
\end{aligned}
$$

Hence, by the asymptotic formula for $\Gamma(x)$, we have $\alpha_{n-k} \geq \alpha_{n-k}^{*}$, where

$$
\alpha_{n-k}^{*} \sim 12 \pi n^{-4} \pi^{k-n-2} \quad \text { as } n \rightarrow \infty,
$$

as desired.

\section{References}

[1] I. Bárany and Z. Füredi, On the shape of the convex hull of random points, Probab. Theory Related Fields 80 (1988), 72-87.

[2] W. Feller, An Introduction to Probability Theory and its Applications, Vol. I, Wiley, New York, 1961.

[3] C. M. Goldie and S. Resnick, Records in a partially ordered set, Ann. Probab. 17 (1989), 678-699.

[4] I. S. Gradshteyn and I. M. Ryzhik, Table of Integrals, Series and Products, Academic Press, New York, 1965.

[5] P. Groeneboom, Limit theorems for convex hulls, Probab. Theory Related Fields 79 (1988), 327-368.

[6] M. G. Kendall, and P. A. P. Moran, Geometrical Probability, Griffin's Statist. Monographs Courses 5, Griffin, London, 1963.

[7] J. F. C. Kingman, Random secants of a convex body, J. Appl. Probab. 6 (1969), $660-672$.

[8] V. B. Nevzorov, Records, Teor. Veroyatnost. i Primenen. 32 (2) (1987), 219-252 (in Russian).

[9] S. Resnick, Extreme Values, Regular Variation, and Point Processes, Springer, Berlin, 1987.

[10] I. M. Yaglom and V. G. Boltyanskiǔ, Convex Bodies, Nauka, Moscow, 1951 (in Russian).

MAREK KAłUSZKA

INSTITUTE OF MATHEMATICS

TECHNICAL UNIVERSITY OF ŁÓDX́

AL. POLITECHNIKI 11

90-924 ŁÓDX́, POLAND 